\title{
Prognostic significance evaluation of B-cell lymphoma 2 (BCL2) and Ki-67 expression in diffuse large B-cell lymphoma patients
}

\author{
Hossein Rahimi ${ }^{\circledR}$, Zahra Rezaei Borojerdi ${ }^{1 *}$, Sajad Ataei Azimi ${ }^{1 *}$, Elnaz Rashidian $^{2^{\circledR}}$, Amirhossein Jafarian $^{3}$ \\ 'Devision of Hematology Oncology, Department of Internal Medicine, Mashhad University of Medical Sciences, Mashhad, Iran \\ ${ }^{2}$ Division of Surgery, Department of Surgery, Mashhad University of Medical Sciences, Mashhad, Iran \\ ${ }^{3}$ Molecular Pathology Research Center, Department of Pathology Faculty, Ghaem Hospital, Mashhad University of Medical Sciences, \\ Mashhad, Iran
}

*Correspondence to Zahra Rezaei Borojerdi, Email: Drzr358@gmail.com and Sajad Ataei Azimi, Email: ataeiazimis@mums.ac.ir

Received 6 Aug. 2019 Accepted 10 Dec. 2019 Published online 25 Dec. 2019

Keywords: Diffuse Large B Cell Lymphoma, Bcl2, Ki-67, Immunohistochemistry

\begin{abstract}
Introduction: Diffuse large B-cell lymphoma (DLBCL) is the most common lymphatic neoplasm, accounting for about $30-40 \%$ of non-Hodgkin's lymphoma cases.

Objectives: DLBCL is a progressive disease with clinical, genetic and molecular heterogeneity. The prognostic value of B-cell lymphoma 2 (BCL2) and Ki-67 in DLBCL patients has been controversial.

Patients and Methods: In this study, we investigated the correlation of BCL2 and Ki-67 expression with clinical features such as age, gender, B symptoms and lactate dehydrogenase (LDH) levels, subtypes of DLBCL, its staging and prognosis in 36 cases of DLBCL. The expression of BCL2 and Ki- 67 was measured by immunohistochemistry.

Results: There was no significant correlation between BCL2 expression and staging ( $P=0.082)$, however Ki-67 expression had a significant correlation with staging $(P=0.002)$. There was no statistically significant correlation between $\mathrm{BCL} 2$ and $\mathrm{Ki}-67$ with prognosis of the disease. We found a significant correlation between the germinal center B-cell $(\mathrm{GCB})$ and non- GCB subtypes with BCL2 expression $(P=0.024)$, since patients with non- GCB subtype had a higher BCL2 expression. Our study also demonstrated a significant relationship between BCL2 and Ki-67 expression, therefore, with the increase of the expression of a marker, another increases $(P=0.045)$. Conclusion: BCL2 and Ki-67 expressions were not associated with prognosis. Overexpression of Ki-67 was associated with higher clinical stages. BCL2 expression is higher in non-GCB subtype of DLBCL. Therefore, our study shows that the subsequent studies of BCL2 and other biomarkers in the DLBCL should be based on the DLBCL subtypes.
\end{abstract}

Citation: Rahimi H, Rezaei Borojerdi Z, Ataei Azimi S, Rashidian E, Jafarian A. Prognostic significance evaluation of B-cell lymphoma 2 (BCL2) and $\mathrm{Ki}-67$ expression in diffuse large B-cell lymphoma patients. Immunopathol Persa. 2020;6(1):e07. DOI:10.15171/ ipp.2020.07.

\section{Introduction}

Diffuse large B-cell lymphoma (DLBCL) is the most common lymphatic neoplasm, accounting for about $30-40 \%$ of nonHodgkin's lymphoma cases (1). DLBCL is a progressive disease, with a heterogeneity at the clinical, genetic and level of expression (2). The diagnosis of DLBCL is based on biopsy of the abnormal lymph node and radiographic imaging. Approximately 50-60\% of patients are treated with a standard chemotherapy regimen with R-CHOP (rituximab, cyclophosphamide, doxorubicin, vincristine, and prednisone) $(3,4)$.

DLBCL based on the gene expression profile, is classified into subtypes of germinal center B-cell like (GCB) with good prognosis, activated B-cell like (ABC) with poor prognosis and $10-15 \%$ of unclassifiable cases (5). Moreover, this disease is mostly classified

\begin{abstract}
Key point
In a retrospective study based on collection of tissue samples of lymph nodes from 36 diffuse large B-cell lymphoma patients, we found a statistically significant correlation between $\mathrm{Ki}-67$ expression and diffuse large B-cell lymphoma stages.
\end{abstract}

into two GCB and non-GCB groups based on Hans's algorithm using multiple myeloma oncogene 1 (MUM1), CD10, and B-cell lymphoma 6 (BCL6) immunohistochemistry (IHC) markers $(3,6)$.

Abnormalities in genes such as BCL2, P53 and MYC have been reported in DLBCL (7). Protein BCL2 is an anti-apoptotic biomarker that plays an important role in the development and differentiation of B cells. The effect of BCL2 expression on survival of patients who received rituximab

\footnotetext{
Copyright $($ C 2020 The Author(s); Published by Nickan Research Institute. This is an open-access article distributed under the terms of the Creative Commons Attribution License (http://creativecommons.org/licenses/by/4.0), which permits unrestricted use, distribution, and reproduction in any medium, provided the original work is properly cited.
} 
is controversial. Some studies have demonstrated that adding rituximab to standard chemotherapy in GCBDLBCL overcomes the poor prognosis of BCL2 expression (8). Although other studies have shown that co-expression of BCL2 and MYC proteins in DLBCL patients have poor prognosis associated with R-CHOP treatment $(9,10)$. In the study by Perry et al, the risk of death in patients with overexpression of BCL2 and MYC was nine times higher compared to down-expression of both biomarkers. Therefore, increased expression of BCL2 and MYC in patients was associated with poor prognosis (11). Ki67 is expressed during the active phase of the cell cycle, especially mitosis, but is not expressed in the G0 phase. Ki-67 has been reported as a marker for identifying nonHodgkin's lymphoma patients with poor prognosis (12). Several studies indicated that overexpression of Ki-67 correlated with poor prognosis in DLBCL patients treated with R-CHOP (13-16).

\section{Objectives}

In this study, the expression of BCL2 and Ki-67 markers in the GCB and non-GCB subtypes of DLBCL was investigated by immunohistochemistry. Then, the association of these markers with the prognosis and clinical stage of the patients has been studied.

\section{Materials and Methods \\ Subjects}

For analysis of BCL2 and Ki-67 expression levels in this descriptive cross-sectional study, we used formalin-fixed, paraffin-embedded tissue samples of the DLBCL patients that admitted in the Ghaem hospital (Mashhad, Iran) in the period from 2007 to 2011. The following samples were excluded from the study; 1) unrepresentative or insufficient tissue sample for the immunohistochemistry analysis; 2) lack of histological confirmation of DLBCL; 3) incomplete documentation in patient clinical records. By applying these exclusion criteria, 36 cases (30 males and 6 females) with DLBCL were analyzed retrospectively.

\section{Immunohistochemistry study}

The paraffin-embedded tissue blocks were cut at 2-5 $\mu \mathrm{m}$ sections. The sections were transferred into glass slides suitable for immunohistochemistry. The slides were allowed to dry at room temperature for 18-24 hours. Then the deparaffinization of slides was conducted in four changes of xylene, ( 5 minutes each). For rehydration, the slides were transferred to $100 \%$ alcohol for 2 minutes and then transfer once through 90\%, 80\% and 70\% alcohols respectively for 3 minutes each. For antigen retrieval, the sections first were incubated in EDTA Tris solution at bain-marie (24-98) for 20 minutes and then rinsed in Tris for $5 \mathrm{~min}$. Blocking endogenous peroxidase activity has done by incubating sections in 3\% $\mathrm{H} 2 \mathrm{O} 2$ solution in methanol at room temperature for 10 minutes and again slides rinsed in Tris for 5 minutes. After Draining off the blocking buffer from the slides, $100 \mu \mathrm{L}$ appropriately diluted primary antibody (Ki-67 and BCL2 antibody dilution buffers are provided ready to use) was applied to the sections on the slides. The sections were then incubated overnight with the BCL2 antibody and for Ki67 incubate in a humidified chamber at room temperature for 1.5 hours. After washing the slides, the sections were then incubated with biotinylated secondary antibody and peroxidase conjugated streptavidin using the Dako Real detection system and diaminobenzidine (Dako) according to the manufacturer's instructions. The final step involved reactivity of the linked enzyme with the chromogen DAB for 10 minutes (Figure 1).

\section{Evaluation}

Immunostaining for Ki-67 and BCL2 was evaluated by determining the number of positive cells expressing nuclear Ki-67 and BCL2 among the total cells using a light microscope. Ki67- was defined as the percentage of total number of tumor cells (at least 1000) with nuclear staining ten randomly selected fields over $10(\times 40)$. Scoring was as follows; (score $\left.1^{+}=0-25 \%\right)$; (score $2^{+}=25-50 \%$ ); (score $\left.3^{+}=50-75 \%\right)$ and (score $\left.4^{+}=75-100 \%\right)$. BCL2 expression was also assessed according to intensity and the scoring was as follows; (score $1^{+}=0-5 \%$ ); (score $2^{+}=5-25 \%$ ); $\left(\right.$ score $\left.3^{+}=25-50 \%\right) ;\left(\right.$ score $\left.4^{+}=50-75 \%\right)$ and (score $5^{+}=$ 75-100 \%). For Ki-67, expression below 25\% was taken as low-expression; the remaining was classified as high and also determined cut off for BCL2 was $25 \%$, therefore, the samples were classified as negative $(<25 \%)$ and positive $(\geq 25 \%)$.

\section{Ethical approval}

The Ethics Committee of Mashhad University of Medical Sciences approved this study (IR.MUI.MED. REC.1398.255043). All study protocols were approved by the institutional ethical committee at Ghaem hospital

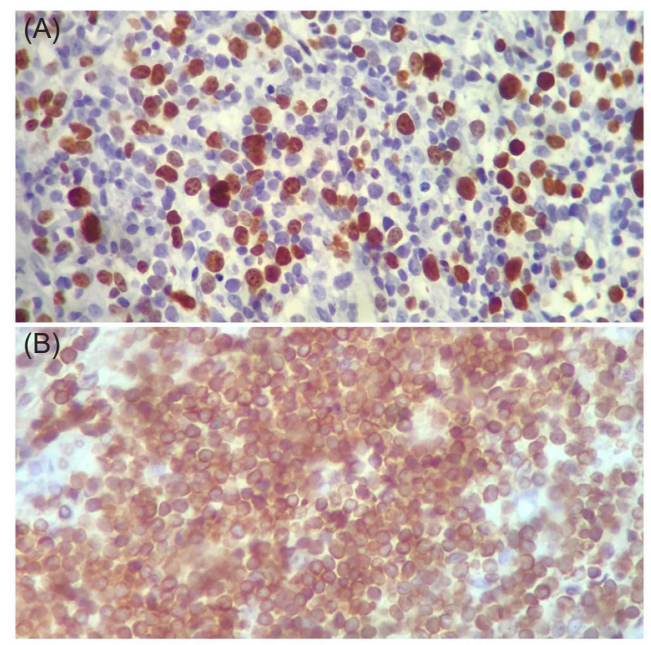

Figure 1. (A) Immunohistochemical staining of nuclei for ki67. Staining is observed in $50 \%$ of cells. (B) Immunohistochemical staining of nuclei for BCL2 $(\times 400)$. 
at Mashhad University of Medical Sciences. Accordingly, written informed consent was taken from all participants before any intervention.

\section{Statistical analysis}

Ki-67 and BCL2 immunopositivity and association between clinicopathological categories were analyzed using the chi-square test, paired $t$ test and Mann-Whitney $\mathrm{U}$ test where applicable. $P$ values of less than 0.05 were considered to indicate the statistical significance. All statistical analyses were carried out by SPSS.

\section{Results}

\section{Patient characteristic}

We designed a retrospective study based on collection of tissue samples of lymph nodes from 36 DLBCL patients since 30 patients $(83.3 \%)$ were male and 6 patients $(16.7 \%)$ were female. The median age of all patients was $46.78 \pm$ 10.75 years (range, $32-71$ years). Among the 36 DLBCL, 20 cases were GCB and 16 were non-GCB. Two patients (5.60\%) were in stage I, 12 patients $(33.30 \%)$ in stage II, 18 patients $(50.00 \%)$ in stage III and 4 patients $(11.10 \%)$ in stage IV of disease. Patient follow up showed that 26 patients $(72.20 \%)$ died and 10 patients $(27.8 \%)$ were alive. All surviving patients were subtypes of GCB. The mean lactate dehydrogenase (LDH) level in all patients was $461.56 \pm 70.12 \mathrm{U} / \mathrm{L}$, which was in men and women 462.53 $\pm 71.43 \mathrm{U} / \mathrm{L}$ and $456.67 \pm 77.68 \mathrm{U} / \mathrm{L}$, respectively. The Mann-Whitney test showed that the median LDH level was similar in men and women $(P=0.999 ; \mathrm{z}=0.99)$.

\section{Relationship between the stages of DLBCL disease with} BCL2 and Ki-67 expression

The expression of BCL2 in 10 patients were $1^{+}(27.8 \%), 6$ patients in $2^{+}(16.7 \%), 4$ patients in $3^{+}(11.1 \%), 14$ patients in $4^{+}(38.9 \%)$ and 2 patients in $5^{+}$in $(5.6 \%)$. While the expression of Ki-67 in 12 patients were $2^{+}(33.3 \%)$, while $3^{+}$ in 6 patients $(16.7 \%)$ and $4^{+}$in 18 patients $(50.0 \%)$. For the expression of BCL2 in immunohistochemical analysis, we have considered cut-off value of $25 \%$ or more as positive (score $3^{+}, 4^{+}$and $5^{+}$) and less than $25 \%$ as negative (score $1^{+}$and $2^{+}$). Table 1 indicates the number of patients and the expression levels of BCL2 and Ki-67 according to the disease stage in the DLBCL. Chi-square tests (linearby-linear association) showed no statistically significant relationship between DLBCL stages and BCL2 expression level $(P=0.082)$. However, a significant difference was observed for Ki-67 expression level with DLBCL stages $(P=0.02)$.

Correlation between expression of BCL2 and Ki-67 with $B$-symptoms, gender and age of patients

Fourteen patients with DLBCL (38.90\%) after 4 years follow-up showed B symptoms and $22(61.10 \%)$ patients had no B symptoms (Table 1). Chi-square test showed no significant correlation between B symptoms with expression of BCL2 and Ki-67 biomarkers $(P>0.05)$. Among 30 male patients, the expression of BCL2 in 6 patients was $1^{+}(16.7 \%)$, in 6 patients was $2^{+}(16.7 \%)$, in 4 patients was $3^{+}(11.1 \%)$, since in 12 patients, it was $4^{+}$ $(33.3 \%)$, and in 2 patient was $5^{+}(5.6 \%)$. Ki-67 was also reported in 10 male patients $2^{+}(27.8 \%)$, in 4 patients $3^{+}(11.1 \%)$ and in 16 patients $4^{+}(44.4 \%)$. No significant correlation was found between gender and age of DLBCL patients with expression of each of BCL2 and Ki-67 markers $(P>0.05)$.

Correlation between expression of BCL2 and Ki-67 with prognosis of disease

Patient follow up showed that 26 patients (72.20\%) died and 10 patients $(27.8 \%)$ were alive (Table 1 ). There was a significant correlation between GCB and non-GCB subtypes with prognostic $(P=0.019)$, therefore the GCB subtype was associated with good prognosis and non-GCB subtype with poor prognosis. All surviving patients were subtypes of GCB, while the expression of BCL2 in four patients was $1^{+}$, in four patients was $2^{+}$, and in two patients, it was $3^{+}$. However, six patients were $2^{+}$for Ki-67 and four

Table 1. Frequency and expression of BCL2 and Ki-67 according to the disease stages, incidence of B symptoms, gender, prognosis, and subtypes of DLBCL patients

\begin{tabular}{|c|c|c|c|c|c|c|c|c|c|}
\hline \multirow{2}{*}{$\begin{array}{l}\text { Clinical } \\
\text { characteristics }\end{array}$} & \multicolumn{5}{|l|}{ BCL2 } & \multicolumn{4}{|l|}{ Ki-67 } \\
\hline & $1^{+}(n)$ & $2^{+}(n)$ & $3^{+}(n)$ & $4^{+}(n)$ & $5^{+}(n)$ & $1^{+}(n)$ & $2^{+}(n)$ & $3^{+}(n)$ & $4^{+}(n)$ \\
\hline Stage I & - & 2 & - & - & - & - & 2 & - & - \\
\hline Stage II & 6 & - & 2 & 4 & - & - & 10 & 2 & - \\
\hline Stage III & 4 & 4 & 2 & 8 & - & - & - & 4 & 14 \\
\hline Stage IV & - & - & - & 2 & 2 & - & - & - & 4 \\
\hline B-symptoms & - & 4 & 2 & 6 & 2 & - & 2 & 2 & 10 \\
\hline Male & 6 & 6 & 4 & 12 & 2 & - & 10 & 4 & 16 \\
\hline Female & 4 & - & - & 2 & - & - & 2 & 2 & 2 \\
\hline Alive & 4 & 4 & 2 & - & - & - & 6 & - & 4 \\
\hline Deceased & 6 & 2 & 2 & 14 & 2 & - & 6 & 6 & 14 \\
\hline GCB & 8 & 6 & 4 & 2 & - & - & 8 & 4 & 8 \\
\hline Non-GCB & 2 & - & - & 12 & 2 & - & 4 & 2 & 10 \\
\hline
\end{tabular}

GCB, germinal center B-cell like. 
others were $4^{+}$. Fisher's exact test showed no significant difference between the prognosis of these patients with the expression of BCL2 or Ki-67 $(P>0.05)$.

Correlation between expression of BCL2 and Ki-67 with subtypes of DLBCL

Among the 36 DLBCL, 20 cases were GCB (55.60\%) and 16 cases were non-GCB (44.40\%). There was a significant correlation between the subtypes of DLBCL and BCL2 expression $(P=0.024)$. Thus, non-GCB subtype patients had higher levels of BCL2 expression (Table 1). However, no significant correlation between DLBCL subtypes and Ki-67 expression was detected $(P=0.638)$.

Correlation between expression of BCL2 with disease stages in subtypes of $G C B$ and non-GCB

For the expression of BCL2 in immunohistochemical analysis, we have considered cut-off value of $25 \%$ or more as positive (score $3^{+}, 4^{+}$and $5^{+}$) and less than $25 \%$ as negative (score $1^{+}$and $2^{+}$). BCL2 expression in GCB was positive in six patients (30\%) and negative in fourteen patients (70\%). Moreover, BCL2 expression in non-GCB was positive in fourteen patients $(87.5 \%)$ and negative in two patients (12.5\%) (Table 2). Fisher's exact test showed no significant difference between the stage of disease and expression of BCL2 in patients with GCB $(P>0.999)$ and non-GCB $(P>0.999)$.

\section{Relationship between expression of Bcl2 and Ki67}

The linear-by-linear association showed a statistically significant relationship between expression of $\mathrm{Bcl} 2$ and Ki67 $(P=0.045)$. Spearman's correlation coefficient for relationship between expression of $\mathrm{Ki} 67$ and $\mathrm{Bcl} 2$ in patients was $0.497(P=0.036)$. Therefore, moderate correlation was observed for these two markers. Therefore, when the value of the expression of a variable (e.g. Ki-67) increases, the value of another variable $(\mathrm{Bcl} 2)$ increases as well (Figure 2).

\section{Discussion}

DLBCL is the most common lymphatic neoplasm, accounting for about $30-40 \%$ of non-Hodgkin's lymphoma cases (1). DLBCL is a progressive disease with clinical, genetic and molecular heterogeneity (2). DLBCL can affect patients of any age, since its incidence is slightly higher in male patients than in women (17). The diagnosis of DLBCL is based on biopsy of the abnormal lymph node and radiographic imaging (3,4). Many clinical, histological, topographical, and demographic factors have been studied in relation to the clinical features and therapeutic responses of the DLBCL, however most of them were insubstantial $(18,19)$.

In this study, we investigated the expression of BCL2 and Ki-67 markers in the GCB and non-GCB subtypes of DLBCL and their relationship with the prognosis and clinical stage of the patients. In our study, no significant
Table 2. BCL2 expression according to subtypes and stages

\begin{tabular}{lllll}
\hline \multirow{2}{*}{ Stage } & \multicolumn{2}{l}{ GCB } & \multicolumn{3}{c}{ Non-GCB } \\
\cline { 2 - 5 } & Positive (n) & Negative (n) & Positive (n) & Negative (n) \\
\hline I & - & 2 & - & - \\
II & 2 & 4 & 4 & 2 \\
III & 4 & 8 & 6 & - \\
IV & - & - & 4 & - \\
\hline
\end{tabular}

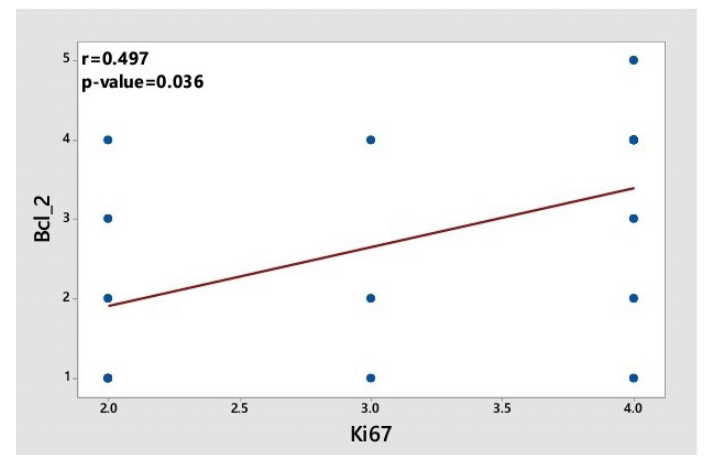

Figure 2. Correlation plot between Ki67 with Bcl2.

difference between expression levels of BCL2 and Ki-67 with age, gender, LDH level and B symptoms was seen. However, in a study by Iqbal et al, a significant difference was shown between BCL2 expression and LDH levels in $\mathrm{ABC}$ subtype patients, while no significant difference was detected regarding clinical characteristics such as age, gender, and LDH in GCB subtype (20). In several other studies, there was no correlation between Ki-67 expression and various clinical features including age, gender, B symptoms, and $\mathrm{LDH}$ was demonstrated $(8,16)$.

In our study, no statistically significant relationship between the stages of DLBCL with expression of BCL2 was seen. However, we found a significant correlation between Ki-67 expression and DLBCL stages, thereby patients with higher Ki-67 expression were at higher clinical stages. However, in several studies no relationship between the expression of Ki-67 and the DLBCL stages was found (15). In the study by Iqbal et al, a correlation between the expression of BCL2 and the stage of the disease only in patients with activated B-cell (ABC) subtype was detected (20).

Several studies have evaluated the expression of specific proteins in DLBCL patients to identify biomarkers for diagnosis, prognosis or outcome $(7,17,21,22)$. Protein BCL2 is an anti-apoptotic biomarker that plays an important role in the development and differentiation of B cells. Overexpression of BCL2 in different subtypes of DLBCL is associated with different mechanisms. In the GCB subtype, transcription $\mathrm{t}(14 ; 18)$ ( $\mathrm{q} 32 ; \mathrm{q} 21$ ) causes overexpression of the BCL2 protein in most cases, whereas in the subtype of ABC, high expression of BCL2 occurs due to gene amplification or pathway activation of NF-KB $(20,23)$. Ki-67 is a nuclear antigen expressed in 
proliferative cells. Expression of this antigen is considered as a potential prognostic biomarker in different DLBCL subtypes. Despite numerous studies to associate the expression of Ki-67 with other biomarkers or therapeutic and clinical outcomes, no definitive conclusion has been made $(16,24,25)$.

In a cohort R-CHOP study by Iqbal et al, the expression of BCL2 had a significant impact on overall survival and event-free survival in the DLBCL and subtype GCBDLBCL but not in the subtype ABC-DLBCL (8). The high expression of BCL2 in the $\mathrm{ABC}$ subtype is related to $18 \mathrm{q} 21$ amplification and pathway activation of NF- $\mathrm{KB}$ $(20,23)$. Rituximab in vitro causes downregulation of NF-кB and its target BCL2 (26), thereby decreasing BCL2 and increasing the sensitivity to chemotherapy. On the other hand, rituximab may not decrease the expression of BCL2 in the subtype of GCB-DLBCL as a result of drug resistance in this subtype of DLBCL (8). In another cohort study, in patients treated with R-CHOP, expression of BCL2 protein had a significant association with overall survival and progression-free survival in GCB-DLBCL (27). Interestingly, in this study, overexpression of BCL2 and Ki-67 markers was significantly associated with decreasing overall survival and progression-free survival in patients with DLBCL, especially in the GCB subtype. Therefore, the use of BCL2 inhibitors may be effective in patients with BCL2 positive GCB-DLBCL. ABT-199 is a selective inhibitor of BCL2, which is effective in patients with DLBCL with BCL2 positive (28). The use of BCL2 selective inhibitors such as ABT-199 may be more effective in patients with GCB-DLBCL (27). The high expression of BCL2 had a significant association with overall survival and progression-free survival in patients with primary central nervous system (CNS)-DLBCL independent of cellular origin (29). In numerous studies, BCL2 has been identified as a poor prognostic factor in patients with DLBCL $(8,11,22,27,30)$. Nevertheless, our results showed no statistically significant relationship between DLBCL prognosis and BCL2 expression. However, this finding could be due to the small sample size in this study. In the study by Iqbal et al, a significant difference between the DLBCL subtypes (GCB and non-GCB) with BCL2 expression was seen, so that patients with non-GCB subtypes had a higher BCL2 expression. Our results are similar to those reported by Iqbal and colleagues, in which BCL2 had a higher expression in the DLBCL with the ABC subtype (20). Therefore, our study clearly shows that the subsequent studies of BCL2 and other biomarkers in the DLBCL should be based on the DLBCL subtypes.

The Ki-67 biomarker has been studied as a useful prognostic index in the DLBCL. Recent studies have shown that overexpression of Ki-67 is a predictor of poor prognosis in patients treated with rituximab (13-16). In addition, high Ki-67 expression was associated with a decrease in overall survival and progression-free survival and higher relapse in patients with DLBCL (15-17). In the study by Jerkeman et al, patients with DLBCL, no significant difference between Ki-67 expression and overall survival in 5 years was presented (31). In several other studies, no clinical relationship between the expression of Ki-67 and DLBCL was seen (7,32). Moreover, in our study, no statistically significant correlation between the expression of Ki-67 with prognosis was seen, which is similar to the results of Perry et al. They found the expression of Ki-67 in GCB and non-GCB subtypes did not show any significant difference (11). Furthermore, in the study of Llanos et al, no association between the high expression of Ki-67 and BCL2 with survival was seen (7). The prognostic value of BCL2 and Ki-67 in DLBCL patients has been controversial. Because there is no consensus to determine the positive and negative levels of these biomarkers, while different cutoff values have been reported in the literature. In addition, a different proportion of GCB and non-GAB patients have been reported in the literature.

Our study also concluded that a significant relationship between BCL2 and Ki-67 expression existed, hence with the increase of the expression of a marker, another marker increases accordingly. This correlation has been shown in the study of Jovanović et al. They showed a significant positive correlation between the high expression of BCL2 and Ki-67 (33). However, in the stud by Yoon et al y, no significant difference was found between Ki-67 and BCL2 expression (15). Interestingly, in our study, a significant relationship between the DLBCL subtypes and the prognosis was seen too. Patients with GCB subtype were associated with better prognosis since non-GCB subtype were with poor prognosis. Similar to our results have been reported in other studies $(5,6,34)$.

\section{Conclusion}

In summary, in our study, no statistically significant relationship between the stages of DLBCL with expression of BCL2 was seen. However, a statistically significant correlation between Ki-67 expression and DLBCL stages was detected, thereby patients with higher Ki-67 expression were at higher clinical stages accordingly. Nevertheless, our results showed no statistically significant relationship between DLBCL prognosis and BCL2 and Ki-67 expression. Of course, this could be due to the small sample size in our study. In this study, a significant difference between the DLBCL subtypes (GCB and non- GCB) with BCL2 expression was shown. Therefore, patients with non- GCB subtypes had a higher BCL2 expression. Our study also demonstrated a significant relationship between BCL2 and $\mathrm{Ki}-67$ expression, therefore with the increase of the expression of a marker, another increases.

\section{Limitations of the study}

The low number of patients may be a limitation for this study. Thus designing a larger study with more participants suggests. 


\section{Authors' contribution}

$H R$ and $A H J$ designed the study. ZRB, and ER performed the experiments and review of the manuscript. ZRB, and SAA analyzed the data. ZRB, HR, AHR, ER, and saa Conducted research and investigation process and data collection wrote the manuscript. All authors approved the final version of the manuscript.

Conflicts of interest

The authors declare no conflicts of interest.

\section{Ethical considerations}

Ethical issues including plagiarism and double publication have been completely observed by the authors.

\section{Funding/Support}

This study was funded by Mashhad University of medical sciences (Grant \# 92/96152).

\section{References}

1. Dunleavy K, Erdmann T, Lenz G. Targeting the B-cell receptor pathway in diffuse large B-cell lymphoma. Cancer Treat Rev. 2018;65:41-6. doi: 10.1016/j.ctrv.2018.01.002.

2. Gascoyne DM, Banham AH. The significance of FOXP1 in diffuse large B-cell lymphoma. Leuk Lymphoma. 2017;58:1037-51. doi: 10.1080/10428194.2016.1228932.

3. Liu Y, Barta SK. Diffuse large B-cell lymphoma: 2019 update on diagnosis, risk stratification, and treatment. Am J Hematol. 2019;94:604-16. doi: 10.1002/ajh.25460.

4. Miao Y, Medeiros LJ, Xu-Monette ZY, Li J, Young KH. Dysregulation of cell survival in diffuse large B cell lymphoma: mechanisms and therapeutic targets. Front Oncol. 2019;9:107. doi: 10.3389/fonc.2019.00107.

5. Friedberg JW, Fisher RI. Diffuse large B-cell lymphoma. Hematol Oncol Clin North Am. 2008;22:941-52. doi: 10.1016/j.hoc.2008.07.002.

6. Hans CP, Weisenburger DD, GreinerTC, Gascoyne RD, Delabie J, Ott G, et al. Confirmation of the molecular classification of diffuse large B-cell lymphoma by immunohistochemistry using a tissue microarray. Blood. 2004;103:275-82.

7. Llanos M, Alvarez-Arguelles H, Aleman R, Oramas J, DiazFlores L, Batista N. Prognostic significance of Ki-67 nuclear proliferative antigen, bcl-2 protein, and p53 expression in follicular and diffuse large B-cell lymphoma. Med Oncol. 2001;18:15-22.

8. Iqbal J, Meyer PN, Smith LM, Johnson NA, Vose JM, Greiner $\mathrm{TC}$, et al. BCL2 predicts survival in germinal center B-cell-like diffuse large B-cell lymphoma treated with CHOP-like therapy and rituximab. Clin Cancer Res. 2011;17:7785-95.

9. Green TM, Young KH, Visco C, Xu-Monette ZY, Orazi A, Go $\mathrm{RS}$, et al. Immunohistochemical double-hit score is a strong predictor of outcome in patients with diffuse large B-cell lymphoma treated with rituximab plus cyclophosphamide, doxorubicin, vincristine, and prednisone. J Clin Oncol. 2012;30:3460-7. doi: 10.1200/JCO.2011.41.4342.

10. Yan LX, Liu YH, Luo DL, Zhang F, Cheng Y, Luo XL, et al. MYC expression in concert with BCL2 and BCL6 expression predicts outcome in Chinese patients with diffuse large B-cell lymphoma, not otherwise specified. PLoS One. 2014;9:e104068. doi: 10.1371/journal.pone.0104068.

11. Perry AM, Alvarado-Bernal Y, Laurini JA, Smith LM, Slack GW, Tan KL, et al. MYC and BCL2 protein expression predicts survival in patients with diffuse large B-cell lymphoma treated with rituximab. Br J Haematol. 2014;165:382-91.

12. Miller TP, Grogan TM, Dahlberg S, Spier CM, Braziel RM, Banks PM, et al. Prognostic significance of the Ki-67associated proliferative antigen in aggressive non-Hodgkin's lymphomas: a prospective Southwest Oncology Group trial. Blood. 1994;83:1460-6.

13. Hasselblom S, Ridell B, Sigurdardottir M, Hansson U, NilssonEhle $\mathrm{H}$, Andersson PO. Low rather than high $\mathrm{Ki}-67$ protein expression is an adverse prognostic factor in diffuse large B-cell lymphoma. Leuk Lymphoma. 2008;49:1501-9. doi: 10.1080/10428190802140055.

14. Szczuraszek K, Mazur G, Jelen M, Dziegiel P, Surowiak P, Zabel M. Prognostic significance of Ki-67 antigen expression in non-Hodgkin's lymphomas. Anticancer Res. 2008;28:11138.

15. Yoon DH, Choi DR, Ahn HJ, Kim S, Lee DH, Kim SW, et al. Ki-67 expression as a prognostic factor in diffuse large B-cell lymphoma patients treated with rituximab plus $\mathrm{CHOP}$. Eur J Haematol. 2010;85:149-57. doi: 10.1111/j.16000609.2010.01467.x.

16. Li ZM, Huang JJ, Xia Y, Zhu YJ, Zhao W, Wei WX, et al. High Ki67 expression in diffuse large B-cell lymphoma patients with non-germinal center subtype indicates limited survival benefit from R-CHOP therapy. Eur J Haematol. 2012;88:510-7. doi: 10.1111/j.1600-0609.2012.01778.x.

17. Patrascu AM, Rotaru I, Olar L, Patrascu S, Ghilusi MC, NeamTu $\mathrm{SD}$, et al. The prognostic role of Bcl-2, Ki67, c-MYC and p53 in diffuse large B-cell lymphoma. Rom J Morphol Embryol. 2017;58:837-43.

18. Rotaru I, Gaman GD, Stanescu C, Gaman AM. Evaluation of parameters with potential prognosis impact in patients with primary gastric diffuse large B-cell lymphoma (PG-DLBCL). Rom J Morphol Embryol. 2014;55:15-21.

19. Saygin C, Jia X, Hill B, Dean R, Pohlman B, Smith MR, et al. Impact of comorbidities on outcomes of elderly patients with diffuse large B-cell lymphoma. Am J Hematol. 2017;92:98996. doi: 10.1002/ajh.24819.

20. Iqbal J, Neppalli VT, Wright G, Dave BJ, Horsman DE, Rosenwald A, et al. BCL2 expression is a prognostic marker for the activated B-cell-like type of diffuse large B-cell lymphoma. J Clin Oncol. 2006;24:961-8. doi: 10.1200/JCO.2005.03.4264.

21. Tsuyama N, Sakata S, Baba S, Mishima $Y$, Nishimura N, Ueda K, et al. BCL2 expression in DLBCL: reappraisal of immunohistochemistry with new criteria for therapeutic biomarker evaluation. Blood. 2017;130:489-500.

22. Riedell PA, Smith SM. Should We Use Cell of Origin and Dual-protein Expression in Treating DLBCL? Clin Lymphoma Myeloma Leuk. 2018;18:91-7. doi: 10.1016/j. clml.2017.12.003.

23. Davis RE, Brown KD, Siebenlist U, Staudt LM. Constitutive nuclear factor kappaB activity is required for survival of activated B cell-like diffuse large B cell lymphoma cells. J Exp Med. 2001;194:1861-74. doi: 10.1084/jem.194.12.1861.

24. Sanchez E, Chacon I, Plaza MM, Munoz E, Cruz MA, Martinez $B$, et al. Clinical outcome in diffuse large B-cell lymphoma is dependent on the relationship between different cell-cycle regulator proteins. J Clin Oncol. 1998;16:1931-9.

25. He X, Chen Z, Fu T, Jin X, Yu T, Liang Y, et al. Ki-67 is a valuable prognostic predictor of lymphoma but its utility varies in lymphoma subtypes: evidence from a systematic meta-analysis. BMC Cancer. 2014;14:153. doi: 10.1186/14712407-14-153.

26. Jazirehi AR, Gan XH, De Vos S, Emmanouilides C, Bonavida B. Rituximab (anti-CD20) selectively modifies Bcl-xL and apoptosis protease activating factor-1 (Apaf-1) expression and sensitizes human non-Hodgkin's lymphoma B cell lines to paclitaxel-induced apoptosis. Mol Cancer Ther. 2003;2:118393.

27. Tang YL, Zhou Y, Cheng LL, Su YZ, Wang CB. BCL2/Ki-67 index predict survival in germinal center B-cell-like diffuse 
large B-cell lymphoma. Oncol Lett. 2017;14:3767-73. doi: 10.3892/ol.2017.6577.

28. Klanova M, Andera L, Brazina J, Svadlenka J, Benesova S, Soukup J, et al. Targeting of BCL2 family proteins with ABT-199 and homoharringtonine reveals BCL2- and MCL1-dependent subgroups of diffuse large B-cell lymphoma. Clin Cancer Res. 2016;22:1138-49.

29. Makino K, Nakamura H, Shinojima N, Kuroda JI, Yano S, Mikami $Y$, et al. BCL2 expression is associated with a poor prognosis independent of cellular origin in primary central nervous system diffuse large B-cell lymphoma. J Neurooncol. 2018;140:115-21. doi:10.1007/s11060-018-2940-3.

30. Li L, Li Y, Que X, Gao X, Gao Q, Yu M, et al. Prognostic significances of overexpression MYC and/or BCL2 in R-CHOPtreated diffuse large B-cell lymphoma: a systematic review and meta-analysis. Sci Rep. 2018;8:6267. doi: 10.1038/s41598018-24631-5.

31. Jerkeman $M$, Anderson $H$, Dictor $M$, Kvaloy $S$, Akerman $M$, Cavallin-Stahl E, et al. Assessment of biological prognostic factors provides clinically relevant information in patients with diffuse large B-cell lymphoma--a Nordic Lymphoma Group study. Ann Hematol. 2004;83:414-9. doi: 10.1007/s00277004-0855-x.

32. Colomo L, Lopez-Guillermo A, Perales M, Rives S, Martinez A, Bosch F, et al. Clinical impact of the differentiation profile assessed by immunophenotyping in patients with diffuse large B-cell lymphoma. Blood. 2003;101:78-84.

33. Jovanovic MP, Jakovic L, Bogdanovic A, Markovic O, Martinovic VC, Mihaljevic B. Poor outcome in patients with diffuse large B-cell lymphoma is associated with high percentage of bcl-2 and Ki 67-positive tumor cells. Vojnosanit Pregl. 2009;66:738-43.

34. Habara T, Sato $\mathrm{Y}$, Takata K, Iwaki N, Okumura H, Sonobe $\mathrm{H}$, et al. Germinal center B-cell-like versus non-germinal center B-cell-like as important prognostic factor for localized nodal DLBCL. J Clin Exp Hematop. 2012;52:91-9. doi: 10.3960/ jslrt.52.91. 